\title{
ORIENTAÇÃO SEXUAL PARA PESSOA COM LESÃO MEDULAR
}

\author{
Marco Antônio Nogueira Magalhães; Juliana Caldas de Souza²; Fernanda Miranda de Oliveira
}

\author{
SEXUAL ORIENTATION FOR PERSON WITH SPINAL CORD INJURY
}

\begin{abstract}
Resumo: É sabido que a lesão medular (LM) pode levar à disfunção sexual na maioria dos casos e que a falta de conhecimento da pessoa portadora de LM, mesmo quando internada em centros de reabilitação, angustia e diminui a autoestima. O presente artigo objetiva conhecer a percepção dos pacientes lesados medulares internados em um centro de reabilitação a respeito da sexualidade, identificar as características sociodemográficas da pessoa com lesão medular portadora de disfunção sexual, verificar o conhecimento do paciente com lesão medular sobre a disfunção sexual, identificar pacientes com ereção reflexa e ereção psicogênica, identificar se houve atividade sexual após a lesão medular, investigar se houve situações ou complicações que interferiram na sexualidade após a lesão medular e quais foram. Trata-se de estudo descritivo, exploratório de abordagem quali-quantitativo. Os participantes foram identificados com a numeração LM-1 a LM-31 de forma impessoal e, a partir da fala de cada um, montou-se quatro subtítulos para apresentação dos resultados encontrados. A LM traz consigo várias mudanças repentinas e desconhecidas pela maioria. Neste estudo, e também em outros tantos consultados, fica evidente que esses pacientes necessitam de instrução quanto às mudanças na sexualidade pós-lesão e que a falta de conhecimento gera ansiedade, medo e frustração, causando no paciente uma visão de ser assexuado e limitado.
\end{abstract}

Palavras-chave: lesão medular; reabilitação; sexualidade

Abstract: It is well known that spinal cord injury can lead to sexual dysfunction in most cases and that the lack of knowledge of the person carrying spinal cord injury, even when hospitalized in anxiety rehabilitation centers and decreases self-esteem. This article aims to know the perception of patients with spinal cord injury hospitalized in a rehabilitation center regarding sexuality, to identify the socio demographic characteristics of the person with spinal cord injury with sexual dysfunction, Identify a patients with reflex erection and psychogenic erection, identify if there was sexual activity after the spinal cord injury, investigate if there were situations or complications that interfered in sexuality after the spinal cord injury and which were. This is a descriptive, exploratory study with a qualitative and quantitative approach. The participants were identified with the numbering LM-1 to LM-31 in an impersonal way and, from the speech of each one, four subheadings were assembled to present the results found. LM brings with it several sudden and unknown changes by the majority. In this study, as well as in many others consulted, it is evident that these patients need instruction regarding changes in post-injury sexuality and that lack of knowledge generates anxiety, fear and frustration, giving the patient a bringing the patient a vision of being asexual and limited.

Key words: spynal cord injury; reabilitation; sexuality

${ }^{1}$ Residente no Programa de Residência Multiprofissional do Centro de Reabilitação e Readaptação Dr. Henrique Santillo, Goiânia, GO, Brasil. E-mail: mnmarco27@hotmail.com

Especialista em Unidade de Terapia Intensiva (UTI), mestra em Enfermagem pela Universidade Federal de Goiás (UFG), tutora em enfermagem no Programa de Residência Multiprofissional da Secretária Estadual de Saúde, responsável técnico do Ambulatório de Enfermagem no Centro de Reabilitação e Readaptação Dr. Henrique Santillo (CRER).

${ }^{3}$ Mestra em Ensino na Saúde pela Universidade Federal de Goiás (UFG), especialista em Unidade de Terapia Intensiva e Controle de Infecção Hospitalar pela Pontifícia Universidade Católica de Goiás (PUC), enfermeira no Centro de Reabilitação e Readaptação Dr. Henrique Santillo, professora na Universidade Alfredo Nasser (UNIFAN) e professora no CEGESP - Centro de Ensino e Pesquisa Goiano, preceptora do Programa de Residência Multiprofissional em Saúde Pública. 


\section{Introdução}

Anualmente a incidência do Trauma Raquimedular se encontra entre 15 a 40 casos por milhão de habitantes mundialmente. Nos Estados Unidos da América (EUA) é encontrada uma incidência de aproximadamente 12 mil novos casos por ano, destes, 4.000 vão a óbito antes mesmo que cheguem ao hospital e outros mil falecem durante o tempo de hospitalização (BRASIL, 2013).

Se tratando de dados estatísticos e epidemiológicos ligados à realidade brasileira, o Brasil está aquém de uma produção bibliográfica necessária para identificar com real clareza a incidência e prevalência da lesão medular (LM) traumática, possivelmente por falta de notificação, o que acarreta dados imprecisos e irreais (op.cit., 2013).

A LM traz consigo grandes mudanças de vida à pessoa, e na grande maioria das vezes são mudanças repentinas que trazem um impacto físico, psicológico, emocional e econômico imensurável, afetando assim, de forma conjunta, o indivíduo e toda a sua família (BORGES, BRIGNOL, SHOELLER, BONETTI, et al., 2012)

Os danos resultantes da LM apresentam-se como perda de movimentos voluntários, da sensibilidade tátil, dolorosa e profunda em membros superiores e inferiores, que vão levar também a alterações no funcionamento dos sistemas respiratório, circulatório, urinário, intestinal, sexual e reprodutivo (SOMERS, 2001).

A maioria dos pacientes, em um primeiro momento, estará preocupado com a melhoria da sua atual condição física. Contudo, quando começam a aceitar a lesão, a sexualidade torna-se um passo importante rumo à reabilitação física e psicológica em todo o processo (WORLDJORTHOP, 2015).

Em um termo mais amplo do que somente a atividade sexual, a sexualidade engloba dimensões físicas, emocionais e socioculturais, além da intimidade, sexo, reprodução, identidade de gênero e papéis, capacidades reprodutivas e orientação sexual (ORGANIZAÇÃO MUNDIAL DA SAÚDE, 2002).

Como a ereção, a ejaculação resulta de uma coordenação psíquica e estimulação sexual física. 0 reflexo ejaculatório tem sua coordenação realizada pela medula espinhal, e esta por sua vez é dependente de fibras simpáticas intactas, presentes na região tóraco-lombar desde os segmentos T10 até L2 e fibras somáticas dos segmentos S2 a S4. O nervo dorsal do pênis é a principal via de ativação por estimulação da glande peniana. Neurônios no córtex, tálamo, hipotálamo e mesencéfalo desempenham papel na ejaculação (CALABRO, POLIMENI, CIUR-
LEO, et al., 2011)

Deste modo, a disfunção ejaculatória pode ser dividida em três partes: ejaculação prematura, ejaculação retardada e ejaculação retrógrada. Dentre essas, a ejaculação retrógrada leva destaque. Essa condição ocorre devido à perda do controle de fechamento e abertura do colo da bexiga e da uretra pelos nervos simpáticos, acarretando acúmulo do ejaculado na bexiga em vez de ser expelido pelo meato uretral (CLEMENT, STÉPHANE, PIERRE, et al., 2013).

O tratamento da disfunção sexual em pacientes com LM objetiva assegurar a qualidade de vida, o retorno da atividade sexual e reprodutiva, além do resgate da autoestima. Um estudo longitudinal evidenciou que após seis meses de LM, os indivíduos se mostraram mais realistas sobre a sua função sexual, e por esse motivo passaram a ter meIhor aceitação da educação sexual (FISHER, BYFIELD, BROWN, FIEDLER, et al., 2001).

Visto isso, se faz necessário conhecer o perfil da pessoa com LM portadora de disfunção sexual em reabilitação, pois permitirá ações de orientação para o cuidado do enfermeiro reabilitador.

\section{Método}

Utilizou-se do método de pesquisa quali-quantitativo. $\mathrm{O}$ método de pesquisa quali-quantitativo pode ser trabalhado em conjunto, pois ambos permitem ver uma análise dialética através de elementos que se complementam, implicando assim uma interpretação mais vasta da realidade colocada (MYNAYO, 2012). A pesquisa foi desenvolvida no Centro de Reabilitação e Readaptação Dr. Henrique Santillo, com 31 pacientes com diagnóstico de LM internados na instituição nos meses de outubro a dezembro de 2016. Para coleta de dados usou-se questionário semiestruturado com questões abertas e fechadas, e após o aceite em participar, solicitou-se a assinatura do Termo de Consentimento Livre e Esclarecido (TCLE) em duas vias. O estudo respeitou os princípios e postulados éticos existentes conforme a Resolução 466 do Conselho Nacional de Saúde (CNS), de 12 de dezembro de 2012. $\mathrm{O}$ projeto foi submetido e aprovado pelo Comitê de Ética do Hospital Geral de Goiânia, conforme número do protocolo CAE 54027216.9.0000.0035 Comitê de Ética do HGG.

\section{Análise dos dados}

Para condução do estudo foi realizada Análise Qualitativa de Bardin, pois a mesma conforma a 
análise do conteúdo em uma forma lógica e contínua por meio de técnicas que são capazes de analisar utilizando-se de procedimentos sistemáticos e objetivos de descrição do conteúdo das mensagens (BARDIN, 2013).

Os resultados se apresentam descritos em cinco categorias: a) aspectos sociodemográficos da população em estudo; b) atividade sexual antes e após a lesão medular; c) conhecimento sobre a disfunção sexual ocasionada pela LM; d) identificação de pacientes com ereção reflexa e psicogênica; e) identificação dos fatores que facilitaram e interferiram na realização do ato sexual.

\section{Resultados}

Aspectos sociodemográficos da população em estudo: Dos 34 pacientes investigados, somente $31(91,1 \%)$ aceitaram participar do estudo, três se recusaram pós-informados dos objetivos. Destes, a predominância foi de pessoas do sexo masculino, sendo eles adultos jovens com uma média de idade de 28,6 anos. Em relação ao tempo de internação para reabilitação variou de 30 a 45 dias.

A média do tempo de LM foi de quatro meses na maioria dos casos. Todos os pacientes (100\%) possuíam lesão aguda, haviam sido encaminhados de serviços especializados pós-estabilização clínica do trauma, momento oportuno para início da reabilitação motora e sexual.
À etiologia da LM destacam-se: acidente de trânsito com nove vítimas (29\%), seguidas de perfuração por arma de fogo (PAF) com oito $(26 \%)$, acidente de moto seis $(19 \%)$, mergulho em águas rasas (mar) três (10\%), queda (ponte, árvore, andaime etc.) três (10\%), infecção (mielite transversa) um (3\%) e acidente cirúrgico um (3\%).

Em relação ao nível medular acometido, $20(65 \%)$ eram paraplégicos, 11 (35\%) eram tetraplégicos. Outro fator importante avaliado diz respeito à relação afetiva desses pacientes, grau de escolaridade e moradia. De acordo com o estudo, oito (26\%) pacientes eram casados, 20 (64\%) eram solteiros e três (10\%) amasiados.

Quanto ao grau de escolaridade, nenhum paciente que participou do estudo tinha ensino superior completo, seis (19\%) tinham ensino superior incompleto, quatro (13\%) tinham ensino médio completo, três (10\%) tinham ensino médio incompleto, cinco (16\%) tinham ensino fundamental completo, oito (26\%), tinham ensino fundamental incompleto, quatro (13\%) foram alfabetizados e um (3\%) era analfabeto.

Com relação à moradia, 18 (58\%) pacientes tinham moradia própria, nove $(29 \%)$ moravam de aluguel e quatro (13\%) a moradia foi cedida. Os dados relacionados ao nível da LM, apresentação e causa da LM estão apresentados na Tabela 1.

\section{Tabela 1 - Nivel da lesào, apresentaçào e a causa da} Lesão Medular.

\begin{tabular}{|l|c|c|}
\hline & Frequência & $\%$ \\
\hline \multicolumn{3}{|c|}{ Diagnóstico Neurofuncional } \\
\hline Paraplegia & $\mathbf{2 0}$ & $\mathbf{6 5} \%$ \\
\hline \multicolumn{2}{|c|}{ Apresentação da LM } \\
\hline \multicolumn{2}{|c|}{ Causa da LM } \\
\hline Completa & $\mathbf{2 5}$ & $\mathbf{8 1} \%$ \\
\hline Incompleta & $\mathbf{6}$ \\
\hline \multicolumn{2}{|c|}{} \\
\hline Acidente de Trânsito & $\mathbf{8}$ & $\mathbf{2 9} \%$ \\
\hline $\begin{array}{l}\text { Perfuração por Arma } \\
\text { de Fogo (PAF) }\end{array}$ & $\mathbf{6}$ & $\mathbf{2 6} \%$ \\
\hline Acidente de Moto & $\mathbf{3}$ & $19 \%$ \\
\hline $\begin{array}{l}\text { Mergulho em águas } \\
\text { rasas (MAR) }\end{array}$ & $\mathbf{3}$ & $\mathbf{1 0} \%$ \\
\hline $\begin{array}{l}\text { Quedas } \\
\text { Infecção }\end{array}$ & $\mathbf{1}$ & $\mathbf{1 0} \%$ \\
Cirúrgico & $\mathbf{3} \%$ \\
\hline
\end{tabular}

Fonte: Os autores, 2017. 
A amostra do estudo em sua maioria é composta de pacientes paraplégicos (65\%), seguida de pacientes tetraplégicos (35\%). O diagnóstico de paraplegia é dado quando a LM ocorre abaixo do nível medular T1 e tetraplegia quando a LM acontece acima desse nível. A apresentação da LM está diretamente relacionada à causa.

Neste estudo e no de Vasconcelos e Riberto (2011), o acidente de trânsito aparece como primeira causa. A PAF como segunda causa é um dado que converge com a literatura descrita, mas demonstra de forma fiel a atual onda de violência vivenciada.

Na Tabela 2, conforme podem ser observados os dados sociodemográficos referentes à escolaridade, estado civil e tipo de moradia, observa-se que a população de menor escolaridade é também a mais acometida, podendo-se inferir que seja um fator de risco existente. É dado comum também a ocorrência da LM em pacientes solteiros e em idade produtiva. Neste estudo $4 \%$ dos pacientes eram solteiros e com vida sexual ativa antes da LM.

Tabela 2 - Dados sociodemográficos sobre escolaridade, estado civil e tipo de moradia.

\begin{tabular}{|c|c|c|}
\hline & Frequència & $\%$ \\
\hline \multicolumn{3}{|c|}{ Grau de Escolaridade } \\
\hline Superior Incompleto & 6 & $19 \%$ \\
\hline Ensino Médio Completo & 4 & $13 \%$ \\
\hline Ensino Médio Incompleto & 3 & $10 \%$ \\
\hline Ensino Fundamental Completo & 5 & $16 \%$ \\
\hline Ensino Fundamental Incompleto & 8 & $26 \%$ \\
\hline Alfabetizado & 4 & $13 \%$ \\
\hline Analfabeto & 1 & $3 \%$ \\
\hline \multicolumn{3}{|c|}{ Estado Civil } \\
\hline Casado & 8 & $26 \%$ \\
\hline Solteiro & 20 & $64 \%$ \\
\hline Amasiado & 3 & $10 \%$ \\
\hline \multicolumn{3}{|c|}{ Moradia } \\
\hline Própria & 18 & $58 \%$ \\
\hline Alugada & 9 & $29 \%$ \\
\hline Cedida & 4 & $13 \%$ \\
\hline
\end{tabular}

Fonte: Os autores, 2017.

\section{Atividade sexual antes e após a Lesão Medular}

Dados do estudo relacionados à atividade sexual antes da LM mostram que três pacientes (10\%) relataram ter relação sexual uma vez na semana, cinco $(16 \%)$ duas vezes na semana, oito $(26 \%)$ três vezes na semana, seis (19\%) quatro vezes na semana, três (10\%) cinco vezes na semana, um (3\%) seis vezes na semana, e cinco (16\%) afirmaram ter mais de seis relações sexual semanalmente.
Pós-LM essa realidade mudou substancialmente. Sete pacientes (22\%) disseram ter atividade sexual uma vez por semana, três $(10 \%)$ duas vezes por semana e 21 (68\%) não tiveram relação pós-lesão. Todos os pacientes referem-se estar insatisfeitos com relação à atividade sexual pós-lesão medular. 
Tabela 3 - Regularidade da atividade sexual antes e após a

Lesão Medular

\begin{tabular}{|c|c|c|c|}
\hline & & Frequência & $\%$ \\
\hline \multirow{2}{*}{ Uma vez na semana } & antes LM & 3 & $10 \%$ \\
\hline & após LM & 7 & $22 \%$ \\
\hline \multirow{2}{*}{$\begin{array}{l}\text { Duas vezes na } \\
\text { semana }\end{array}$} & antes LM & 5 & $16 \%$ \\
\hline & após LM & 3 & $10 \%$ \\
\hline \multirow{2}{*}{$\begin{array}{l}\text { Três vezes na } \\
\text { semana }\end{array}$} & antes LM & 8 & $26 \%$ \\
\hline & após LM & 0 & $0 \%$ \\
\hline \multirow{2}{*}{$\begin{array}{l}\text { Quatro vezes na } \\
\text { semana }\end{array}$} & antes LM & 6 & $19 \%$ \\
\hline & após LM & 0 & $0 \%$ \\
\hline \multirow{2}{*}{$\begin{array}{l}\text { Cinco vezes na } \\
\text { semana }\end{array}$} & antes LM & 3 & $10 \%$ \\
\hline & após LM & 0 & $0 \%$ \\
\hline \multirow{2}{*}{$\begin{array}{l}\text { Seis vezes na } \\
\text { semana }\end{array}$} & antes LM & 1 & $3 \%$ \\
\hline & após LM & 0 & $0 \%$ \\
\hline \multirow{2}{*}{$\begin{array}{l}\text { Mais que seis vezes } \\
\text { na semana }\end{array}$} & antes LM & 5 & $16 \%$ \\
\hline & após LM & 0 & $0 \%$ \\
\hline \multirow{2}{*}{ Não teve } & antes LM & 0 & $0 \%$ \\
\hline & após LM & 21 & $86 \%$ \\
\hline
\end{tabular}

Fonte: Os autores, 2017.

\section{Conhecimento sobre a disfunção sexual ocasionada pela LM}

Os dados referentes ao conhecimento dos pacientes do estudo sobre a disfunção sexual ocasionado pela LM mostram conhecimento deficiente de que a LM é capaz de agir de forma negativa sobre a sexualidade, como mostram os trechos abaixo retirados do questionário.

[...] Não sabia que o acidente causava isso porque ninguém até o momento havia me informado, achava que não tinha relação uma coisa com a outra. (LM-18, tetraplegia C6 por PAF)

[...] Não sabia que isso acontecia depois do acidente. Preciso muito de ajuda, sou casado. (LM15, tetraplegia C6 por acidente de moto)

[...] Não me sinto satisfeito sexualmente. Não sabia que isso era por causa da lesão. Incomoda sim, quero ajuda. (LM-15, paraplegia T3 por acidente de moto)

[...] Não sabia até então que isso é devido à lesão. Gostaria muito que alguém me esclarecesse, porque incomoda. (LM-14, Paraplegia L1-L2 por queda.)

[...] Não sabia que o acidente causava isso. Incomoda-me muito saber, já estava sentindo a diferença. (LM-24, paraplegia T7por automobilístico)

Identificando pacientes com ereção reflexa e psicogênica
Para identificação dos pacientes com ereção reflexa e psicogênica dois fatores foram essenciais: a altura da lesão e também perguntas sobre o que de fato os deixava excitados (as).

[...] Tenho ereção psicogênica, sei porque sempre que olho pra minha esposa pelada sinto vontade de fazer sexo e de outra forma meu pênis não fica duro. (LM-6, paraplégico L1 por PAF).

[...] Meu pênis fica duro quando pega, inclusive quando passa a sonda para tirar o xixi, eu acho que é reflexa. (LM-3 paraplegia T7completa por queda).

[...] Acho que minha ereção é reflexa, porque sempre que pega no meu pênis ele fica duro, até mesmo quando faz o CVI (cateterismo vesical intermitente). (LM-1, tetraplégico C6-C7 por acidente automobilístico)

[...] Sem dúvida a minha ereção é psicogênica, porque já me falaram em outro hospital e também aonde foi a lesão. (LM-5, paraplégico L1 lesão por PAF)

[...] A ereção acontece quando olho a minha esposa pelada e também quando assisto filmes adultos, por isso acho que é psicogênica. (LM-14 L1-L2 por queda)

[...] Mesmo após explicação acho que fico excitada de forma psicogênica, mas a minha vagina não molha e não tive mais menstruação. (LM-22, paraplegia T10 por PAF) 
Tabela 4 - Tipos de ereção pós-lesão medular

\begin{tabular}{|c|c|c|}
\hline \multicolumn{3}{|c|}{ Tipo de Ereção } \\
\hline Psicogênica & 6 & $19 \%$ \\
\hline Reflexa & 25 & $81 \%$ \\
\hline
\end{tabular}

Fonte: Os autores, 2017.

Como colocado, a ocorrência de ereção psicogênica ou reflexa está intimamente relacionada ao nível medular acometido. Observamos que $19 \%$ dos pacientes relataram ter ereção psicogênica e $81 \%$ ereção reflexa pós-orientação, porém foi relato comum entre esses a ereção insatisfatória, causando frustração e revolta.

Identificando os fatores que facilitaram e interferiram na realização do ato sexual pós-lesão

Quando passada a fase de luto sexual, o LM se depara com um dos maiores dilemas enfrentados, principalmente para pessoa do sexo masculino. São vários os fatores que podem interferir de forma positiva ou negativa na realização do ato sexual, gerando assim mais ansiedade e frustração quando algo sai inconveniente. Diante das falas dos pacientes percebe-se o quanto precisam de ajuda para passar por essa realidade.

[...] O medo, a ansiedade e falta de privacidade foram os principais fatores que interferiram na realização do ato sexual. (LM-1, tetraplegia C6-C7 completa por acidente automobilístico)

[...] Não teve nenhum fator que facilitou. Tentei fazer quando estava internado, mas foi ruim, o pênis não fica duro o tempo todo. (LM-4, paraplegia T11 completa por PAF)

[...]. O fator que interferiu foi a falta de tempo, falta de privacidade, morando com parentes. Não teve fator que facilitou. (LM-5, paraplegia L1 completa por PAF)

[...] Um fator que interferiu muito foi o tempo e a minha esposa estava muito cansada. Não identifico fator facilitador doutor, porque ainda não tentei, não fui pra casa ainda. (LM11, tetraplegia C4 por MAR)

[...] Depois que fui pra casa tentamos al- gumas vezes, o que dificultou foram as posições, a força de vontade foi o que facilitou. (LM-13, paraplegia T6 completa por PAF)

\section{Discussão}

A prevalência da LM por acidente de trânsito aparece em todos os estudos analisados. $O$ estudo de Custodio et al., (2009) demonstra que o acidente de trânsito aparece como primeira causa $(44,70 \%)$ seguido de acidentes por motocicleta $(17,78 \%)$, corroborando com o presente estudo. Uma busca na literatura demonstra que o envolvimento em acidentes de trânsito advém de fatores de risco como a falta de habilitação e a idade do condutor (ZAKRAJSEK, SHOPE, 2006).

Corrobora também com o estudo realizado em 2013, pela Organização Mundial da Saúde (OMS), que destacou o acidente de carro, a queda de grandes alturas e a violência como $90 \%$ das causas de LM (OMS, 2013). Outro dado importante encontrado neste estudo diz respeito à PAF como segunda causa da LM. Goiás aparece em quarto lugar entre os estados com mais vítimas por homicídio e o sexto com mais mortes envolvendo armas de fogo, podendo estar associado ao aumento da incidência de casos de LM pós-PAF (MAPA DA VIOLÊNCIA, 2016).

Quando analisamos o grau de escolaridade, percebemos, neste estudo e em outros, que a LM está mais presente em pacientes com grau de escolaridade mais baixa. $\mathrm{O}$ estudo de Trierveiler et al., (2015), com 45 pacientes, demonstrou que seis tinham ensino superior completo, 12 ensino superior incompleto, 10 ensino médio completo, um ensino médio incompleto, dois ensino fundamental completo e 14 fundamental incompleto. No mesmo estudo, 34 pacientes tinham moradia própria, oito moradias alugadas, três pacientes possuíam moradias cedidas. Dados esses que vão de encontro ao estudo em questão.

Analisar a regularidade da atividade sexual semanalmente dá uma visão geral do quanto a sexualidade do paciente LM é afetada. Abdo et al. (2002) demonstrou que os brasileiros relatam fazer 
sexo, em média, 2,9 vezes por semana. Neste estudo, sete $(22 \%)$ pacientes disseram ter regularidade sexual uma vez na semana pós-lesão, três (10\%) relataram ter relação duas vezes na semana e 21 (68\%) não tiveram relação sexual pós LM.

Essa realidade diferencia-se da encontrada por Torrecilha et al. (2014) no trabalho intitulado: o perfil da sexualidade em homens com lesão medular, onde foi observado que de 36 pacientes, $75 \%$ mantiveram vida sexual ativa pós lesão medular, sendo que destes, $16 \%$ tinham relação sexual uma vez por semana. Infere-se que, essa diferença seja devido à condição de cada paciente, em cada pesquisa, visto que neste estudo, os pacientes estavam na fase aguda da lesão e hospitalizados, fatores os quais acreditamos que interferem negativamente para que $o$ ato sexual aconteça.

Torrecilha et al. (2014) descreveram que de 36 pacientes 34 tinham desejo sexual preservado pós-lesão, sendo que 29 eram satisfeitos sexualmente e sete insatisfeitos. $O$ tempo de LM desses pacientes variou de sete a nove anos. Biering-Sorensen et al. (2012) afirmaram que 70 a $80 \%$ dos indivíduos jovens com LM com mais de seis meses apresentam maior facilidade para apresentar ereção, tendo, portanto, maior facilidade no ato sexual e melhora na satisfação.

Esses estudos demonstram o quanto o tempo de LM é fator determinante para melhora da função sexual do lesado medular. Dentre os fatores que interferiram no ato sexual no presente estudo, a hospitalização e a falta de privacidade são os mais citados, diferente dos fatores encontrados por Torrencilha (2014) em que o prejuízo da sensibilidade, a mobilidade reduzida, a não ocorrência do orgasmo e a baixa autoestima foram os mais descritos.

A orientação deficiente pode ser vista nas falas dos participantes do estudo, não sendo fator isolado. Evidencia-se que os profissionais da saúde que trabalham com reabilitação tendem a não transmitirem a orientação sexual aos pacientes vítimas de LM, fator suficiente para que ocorram mais investimentos e capacitação. Os pacientes, quando não orientados, sentem vergonha de perguntar e acabam sofrendo, devido ao desconhecimento de que forma podem voltar a ter uma prática sexual, gerando falta de interesse, sendo então deixada de lado (FARO, 1991).

Logo no primeiro contato com o paciente lesado medular devem ser aprofundados os conhecimentos sobre a sexualidade humana e sua imensa diversidade, voltando-se para a fisiologia, tendo em vista que profissionais bem capacitados contribuem significativamente na reabilitação social e sexual
(BAASCH, 2008).

Exercitar a resiliência desses pacientes é parte importante do cuidado dos profissionais, pois se abstrai dos mesmos pontos positivos de enfrentamento à nova condição gerada, colocando em destaque adjetivos pessoais capazes de gerar melhora da qualidade de vida (SHOELLER, 2012).

\section{Conclusão}

A Lesão Medular traz consigo várias mudanças repentinas e desconhecidas pela maioria. Fica evidente neste estudo que essas pessoas necessitam de instrução quanto às mudanças na sexualidade pós-Lesão Medular e que a falta de conhecimento pode provocar ansiedade, medo e frustração, trazendo uma visão de ser assexuado e limitado.

O não entendimento do parceiro ou da parceira sobre as mudanças ocorridas e como contorná-las tende a levar ao desgaste no relacionamento e por consequência o rompimento, sendo essa uma das tantas perdas que a pessoa com LM terá que conviver.

O sexo é considerado tabu, motivo pelo qual até mesmo os profissionais envolvidos no cuidado não se sentem confortáveis para falar sobre o tema. O enfermeiro é o profissional que passa a maior parte do tratamento ao lado do paciente, muitas vezes na fase aguda da lesão, devendo estar apto a conhecer as mudanças corporais e sexuais para só então definir o seu plano de cuidados.

\section{Referências}

ABDO C.H.N, OLIVEIRA W.M, FITTIPALDI J.A.S. Perfil sexual da população brasileira: resultados do estudo comportamental sexual (ECOS) do brasileiro. Rev. Bras. Med., v. 59, n. 4, p. 250-7, 2002.

BAASCH, A.K.M. Sexualidade na lesão medular. Dissertação (mestrado), Florianópolis, Universidade do Estado de Santa Catarina. 267 f, 2008

BARDIN, L. Análise de conteúdo. Lisboa: Edições 70, 2013.

BIERING-SORENSEN, I.; HANSEN, R. B.; BIERING-SORENSEN, F. Sexual function in a traumatic spinal cord injured population 10-45 years after injury. J. Rehabil. Med. v. 44, n. 11, p. 926-931, 2012.

BORGES, A. M. F; BRIGNOL, P; SCHOELLER, S. D; BONETTI, A. Percepção das pessoas com lesão medular sobre a sua condição. ver. Gaúcha En- 
ferm n. 3, v. 33, pag. 119-125, 2012. Disponível em: http://www.scielo.br/scielo.phpscriptsciarttext \&pidS198314472012000300016>. Acesso em: 04 set. 2016.

BRASIL. Diretrizes de atenção às pessoas com Lesão Medular. Disponível em: http://bvsms.saude. gov.br/bvs/publicacoes/diretrizes_atencao_pessoa_ lesao_medular. Pdf, 2013. Acesso em: 10 ago. 2016.

CALABRO, R. S.; POLIMENI, G.; CIURLEO, R.; CASELLA, C.; BRAMANTI, P. Distúrbios ejaculatórios. Neurogênicas: foco em tratamentos actuais e futuros. Recentes Pat CNS Drogas Discov, v. 6, p. 205-21, 2011.

CLEMENT, C.; STÉPHANE, B.; PIERRE, D.; PIERRE, C.; JACQUES, B.; FRANÇOIS, G. The spinal controlo of ejaculation revised: a systematic review and meta-analysis of anejaculation in spinal cord injured patients. Human reproduction update, v. 19, n. 5, p. 507-526, 2013.

CUSTODIO, N. R. O.; CARNEIRO, M. R.; FERES, C. C.; LIMA, G. H. S.; JUBE, M. R. R.; WATANABE, L. E.; SALIBA, L. G. R. S. O.; DAHER, S.; GARCIA, A. C. F. Lesão Medular no Centro de Reabilitação Dr. Henrique Santillo (CRER-GO). Coluna/Columna, v. 8, n. 3, p. 265-268, 2009.

FARO, A.C.M. Estudo das alterações da função sexual em homens paraplégicos. Dissertação (Mestrado). Escola de Enfermagem da Universidade de São Paulo, 1991.

FISHER, T.; BYFIELD, M. G.; BROWN, T. T.; FIEDLER, I.; LAUD, P. O perfil de necessidades de saúde sexual dos indivíduos de 12 meses após a lesão medular. SCI Psychosoc Proc, v. 14 n. 1, p. 5-11, 2001.

MINAYO, M.C. S.; GOMES, S. F. Pesquisa social: teoria, método e criatividade. 32. ed. Petrópoles: Vozes, 2012.

ORGANIZAÇÃO MUNDIAL DA SAÚDE. Relatório mundial da saúde. Saúde mental: nova concepção, nova esperança. Lisboa, ed. 1, abril de 2002.

RIBERTO, M.; VASCONCELOS, M. L. C. E. Caracterização clínica das situações de fratura da coluna vertebral no município de Ribeirão Preto, propostas para um programa de prevenção do trauma raquimeduar. Coluna/Columna, v. 10, n. 1, p. 40-3, 2011.
SCHOELLER, S.D. Mudanças na qualidade de vida das pessoas com lesão medular adquirida. Rev. Eletr. Goiânia, v 14, p 95-103, jan/mar, 2012. Disponível em: <https: // www.fen.ufg.br/fen_revista/v14/ n1/pdf/v14n1a11.pdf:> Acesso em: 22 dez. 2016.

SOMERS, M.F. Spinal cord injury: functional rehabilitation. 2. ed. New Jersey: Prentice - Hall, 2001. $458 \mathrm{p}$.

TORRECILHA, L. A.; COSTA, B. T.; LIMA, B. F.; SANTOS, S. M. S.; SOUZA, B. R. O perfil da sexualidade em homens com lesão medular. Fisioter. Mov, Curitiba, v. 27, n. 1, p. 39-48, jan.-mar, 2014.

TRIERVEILER, K. S.; RAMOS, F. R. S.; SCHOELLER, S. D.; NOGUEIRA, G. C.; MARTINS, M. M. F. P.S.; SCHNEIDER, D. G. Funcionalidade familiar da pessoa com lesão medular. Texto Contexto Enferm. Florianópolis, v. 24, n. 4, p. 993-1002, out.-dez. 2015.

ZAKARAJSEK, J. S.; SHOPE, J. T. Longitudinal examination of underage drinking and subsequente drinking and risky driving. Joirnal of Safety Research, v. 37, p. 443-451, 2006. 